\title{
Inverse Perron values and connectivity of a uniform hypergraph
}

\author{
Changjiang $\mathrm{Bu}^{*}$ \\ College of Automation \\ College of Science \\ Harbin Engineering University \\ Harbin, PR China \\ buchangjiang@hrbeu.edu.cn
}

\author{
Haifeng Li \\ College of Automation \\ Harbin Engineering University \\ Harbin, PR China \\ lihaifeng0820@163.com
}

\author{
Jiang Zhou \\ College of Science \\ Harbin Engineering University \\ Harbin, PR China \\ zhoujiang04113112@163.com
}

Submitted: Oct 21, 2017; Accepted: Oct 21, 2018; Published: Nov 2, 2018

(C) The authors. Released under the CC BY-ND license (International 4.0).

\begin{abstract}
In this paper, we show that a uniform hypergraph $\mathcal{G}$ is connected if and only if one of its inverse Perron values is larger than 0 . We give some bounds on the bipartition width, isoperimetric number and eccentricities of $\mathcal{G}$ in terms of inverse Perron values. By using the inverse Perron values, we give an estimation of the edge connectivity of a 2-design, and determine the explicit edge connectivity of a symmetric design. Moreover, relations between the inverse Perron values and resistance distance of a connected graph are presented.
\end{abstract}

Mathematics Subject Classifications: 05C50, 05C65, 05C40, 05C12, 15A69

\section{Introduction}

Let $V(\mathcal{G})$ and $E(\mathcal{G})$ denote the vertex set and edge set of a hypergraph $\mathcal{G}$, respectively. $\mathcal{G}$ is $k$-uniform if $|e|=k$ for each $e \in E(\mathcal{G})$. In particular, 2-uniform hypergraphs are usual graphs. For $i \in V(\mathcal{G}), E_{i}(\mathcal{G})$ denotes the set of edges containing $i$, and $d_{i}=\left|E_{i}(\mathcal{G})\right|$

\footnotetext{
*Corresponding author. This work is supported by the National Natural Science Foundation of China (No. 11371109, No. 11601102 and No. 11671108), the Fundamental Research Funds for the Central Universities (No. GK2110260149).
} 
denotes the degree of $i$. The adjacency tensor [8] of a $k$-uniform hypergraph $\mathcal{G}$, denoted by $\mathcal{A}_{\mathcal{G}}$, is an order $k$ dimension $|V(\mathcal{G})|$ tensor with entries

$$
a_{i_{1} i_{2} \cdots i_{k}}= \begin{cases}\frac{1}{(k-1) !}, & \text { if }\left\{i_{1}, i_{2}, \ldots, i_{k}\right\} \in E(\mathcal{G}), \\ 0, & \text { otherwise. }\end{cases}
$$

The Laplacian tensor [27] of $\mathcal{G}$ is $\mathcal{L}_{\mathcal{G}}=\mathcal{D}_{\mathcal{G}}-\mathcal{A}_{\mathcal{G}}$, where $\mathcal{D}_{\mathcal{G}}$ is the diagonal tensor of vertex degrees of $\mathcal{G}$. Recently, the research on spectral hypergraph theory via tensors has attracted much attention $[7-10,14,19,24]$. The spectral properties of the Laplacian tensor of hypergraphs are studied in $[13,25,27,29,35]$.

For an order $k$ dimension $n$ tensor $\mathcal{T}=\left(t_{i_{1} i_{2} \cdots i_{k}}\right)$, let $\mathcal{T} \mathbf{x}^{k}=\sum_{i_{1}, \ldots, i_{k}=1}^{n} t_{i_{1} i_{2} \cdots i_{k}} x_{i_{1}} \cdots x_{i_{k}}$. The algebraic connectivity of a graph plays important roles in spectral graph theory [11]. Analogue to the algebraic connectivity of a graph, Qi [27] defined the analytic connectivity of a $k$-uniform hypergraph $\mathcal{G}$ as

$$
\alpha(\mathcal{G})=\min _{j=1, \ldots, n} \min \left\{\mathcal{L}_{\mathcal{G}} \mathbf{x}^{k}: \mathbf{x} \in \mathbb{R}_{+}^{n}, \sum_{i=1}^{n} x_{i}^{k}=1, x_{j}=0\right\},
$$

where $n=|V(G)|, \mathbb{R}_{+}^{n}$ denotes the set of nonnegative vectors of dimension $n$. Qi proved that $\mathcal{G}$ is connected if and only if $\alpha(\mathcal{G})>0$. In [20], some bounds on $\alpha(\mathcal{G})$ were presented in terms of degree, vertex connectivity, diameter and isoperimetric number. A feasible trust region algorithm of $\alpha(\mathcal{G})$ was given in [9].

For any vertex $j$ of a $k$-uniform hypergraph $\mathcal{G}$, we define the inverse Perron value of $j$ as

$$
\alpha_{j}(\mathcal{G})=\min \left\{\mathcal{L}_{\mathcal{G}} \mathbf{x}^{k}: \mathbf{x} \in \mathbb{R}_{+}^{n}, \sum_{i=1}^{n} x_{i}^{k}=1, x_{j}=0\right\} .
$$

Clearly, the analytic connectivity $\alpha(\mathcal{G})=\min _{j \in V(\mathcal{G})} \alpha_{j}(\mathcal{G})$ is the minimum inverse Perron value. For a connected graph $G, \alpha_{j}(G)$ is the minimum eigenvalue of $\mathcal{L}_{G}(j)$, where $\mathcal{L}_{G}(j)$ is the principal submatrix of $\mathcal{L}_{G}$ obtained by deleting the row and column corresponding to $j$. $\mathcal{L}_{G}(j)$ is nonsingular and its inverse $\mathcal{L}_{G}(j)^{-1}$ is a nonnegative matrix [16]. It is easy to see that $\alpha_{j}^{-1}(G)$ is the spectral radius of $\mathcal{L}_{G}(j)^{-1}$, which is called the Perron value of $G$. All inverse Perron values of a tree $T$ can determine the algebraic connectivity of $T$ $[1,15]$.

The resistance distance $[17,34]$ is a distance function on graphs. For two vertices $i, j$ in a connected graph $G$, the resistance distance between $i$ and $j$, denoted by $r_{i j}(G)$, is defined to be the effective resistance between them when unit resistors are placed on every edge of $G$. The Kirchhoff index $[17,33]$ of $G$, denoted by $K f(G)$, is defined as the sum of resistance distances between all pairs of vertices in $G$, i.e., $K f(G)=\sum_{\{i, j\} \subseteq V(G)} r_{i j}(G) . K f(G)$ is a global robustness index. The resistance distance and Kirchhoff index in graphs have been investigated extensively in mathematical and chemical literatures $[3,4,6,12,23,31,36]$. 
This paper is organized as follows. In Section 2, some auxiliary lemmas are introduced. In Section 3, we show that a uniform hypergraph $\mathcal{G}$ is connected if and only if one of its inverse Perron values is larger than 0 , and some inequalities among the inverse Perron values, bipartition width, isoperimetric number and eccentricities of $\mathcal{G}$ are established. Partial results improve some bounds in [20, 27]. We also use the inverse Perron values to estimate the edge connectivity of 2-designs. In Section 4, some inequalities among the inverse Perron values, resistance distance and Kirchhoff index of a connected graph are presented.

\section{Preliminaries}

For a positive integer $n$, let $[n]=\{1,2, \ldots, n\}$. An order $m$ dimension $n$ tensor $\mathcal{T}=$ $\left(t_{i_{1} \cdots i_{m}}\right)$ consists of $n^{m}$ entries, where $i_{j} \in[n], j \in[m]$. When $m=2, T$ is an $n \times n$ matrix. Let $\mathbb{R}^{[m, n]}$ denote the set of order $m$ dimension $n$ real tensors, and let $\mathbb{R}_{+}^{n}$ denote the cone of nonnegative vectors in $\mathbb{R}^{n}$. For $\mathcal{T}=\left(t_{i_{1} i_{2} \cdots i_{m}}\right) \in \mathbb{R}^{[m, n]}$ and $\mathbf{x}=\left(x_{1}, \ldots, x_{n}\right)^{\mathrm{T}} \in \mathbb{R}^{n}$, let $\mathcal{T} \mathbf{x}^{m-1} \in \mathbb{R}^{n}$ denote the vector whose $i$-th component is

$$
\left(\mathcal{T} \mathbf{x}^{m-1}\right)_{i}=\sum_{i_{2}, i_{3}, \ldots, i_{m}=1}^{n} t_{i i_{2} \cdots i_{m}} x_{i_{2}} x_{i_{3}} \cdots x_{i_{m}}
$$

and let $\mathbf{x}^{[m-1]}=\left(x_{1}^{m-1}, \ldots, x_{n}^{m-1}\right)^{\mathrm{T}}$. In 2005, Qi [26] and Lim [21] proposed the concept of eigenvalues of tensors, independently. For $\mathcal{T}=\left(t_{i_{1} i_{2} \cdots i_{m}}\right) \in \mathbb{R}^{[m, n]}$, if there exist a number $\lambda \in \mathbb{R}$ and a nonzero vector $\mathbf{x}=\left(x_{1}, \ldots, x_{n}\right)^{\mathrm{T}} \in \mathbb{R}^{n}$ such that $\mathcal{T} \mathbf{x}^{m-1}=\lambda \mathbf{x}^{[m-1]}$, then $\lambda$ is called an $H$-eigenvalue of $\mathcal{T}$, $\mathbf{x}$ is called an $H$-eigenvector of $\mathcal{T}$ corresponding to $\lambda$.

For a vertex $j$ of a $k$-uniform hypergraph $\mathcal{G}$, let $\mathcal{L}_{\mathcal{G}}(j) \in \mathbb{R}^{[k, n-1]}$ denote the principal subtensor of $\mathcal{L}_{\mathcal{G}} \in \mathbb{R}^{[k, n]}$ with index set $V(\mathcal{G}) \backslash\{j\}$. By Lemma 2.3 in [32], we know that $\alpha_{j}(\mathcal{G})$ is the smallest H-eigenvalue of $\mathcal{L}_{\mathcal{G}}(j)$ for any $j \in V(\mathcal{G})$.

A path $\mathcal{P}$ of a uniform hypergraph $\mathcal{G}$ is an alternating sequence of vertices and edges $v_{0} e_{1} v_{1} e_{2} \cdots v_{l-1} e_{l} v_{l}$, where $v_{0}, \ldots, v_{l}$ are distinct vertices of $\mathcal{G}, e_{1}, \ldots, e_{l}$ are distinct edges of $\mathcal{G}$ and $v_{i-1}, v_{i} \in e_{i}$, for $i=1, \ldots, l$. The number of edges in $\mathcal{P}$ is the length of $\mathcal{P}$. For all $u, v \in V(\mathcal{G})$, if there exists a path starting at $u$ and terminating at $v$, then $\mathcal{G}$ is said to be connected [5].

Lemma 1. [27] The uniform hypergraph $\mathcal{G}$ is connected if and only if $\alpha(\mathcal{G})>0$.

Let $\mathcal{G}$ be a $k$-uniform hypergraph, $S \neq \emptyset$ be a proper subset of $V(\mathcal{G})$. Denote $\bar{S}=$ $V(\mathcal{G}) \backslash S$. The edge-cut set $E(S, \bar{S})$ consists of edges whose vertices are in both $S$ and $\bar{S}$. The minimum cardinality of such an edge-cut set is called edge connectivity of $\mathcal{G}$, denote by $e(\mathcal{G})$.

Lemma 2. [27] Let $\mathcal{G}$ be a k-uniform hypergraph with $n$ vertices. Then

$$
e(\mathcal{G}) \geqslant \frac{n}{k} \alpha(\mathcal{G})
$$


The $\{1\}$-inverse of a matrix $M$ is a matrix $X$ such that $M X M=M$. Let $M^{(1)}$ denote any $\{1\}$-inverse of $M$, and let $(M)_{i j}$ denote the $(i, j)$-entry of $M$.

Lemma 3. [2, 34] Let $G$ be a connected graph. Then

$$
r_{i j}(G)=\left(\mathcal{L}_{G}^{(1)}\right)_{i i}+\left(\mathcal{L}_{G}^{(1)}\right)_{j j}-\left(\mathcal{L}_{G}^{(1)}\right)_{i j}-\left(\mathcal{L}_{G}^{(1)}\right)_{j i} .
$$

Let $\operatorname{tr}(A)$ denote the trace of the square matrix $A$, and let $\mathbf{e}$ denote an all-ones column vector.

Lemma 4. [30] Let $G$ be a connected graph of order $n$. Then

$$
K f(G)=n \operatorname{tr}\left(\mathcal{L}_{G}^{(1)}\right)-\mathbf{e}^{\top} \mathcal{L}_{G}^{(1)} \mathbf{e} .
$$

Lemma 5. [2] Let $G$ be a connected graph with $n$ vertices and $i \in[n]$. Let $\mathcal{L}_{G}=$ $\left(\begin{array}{ccc}L_{1} & \mathbf{x} & L_{2} \\ \mathbf{x}^{\mathrm{T}} & d_{i} & \mathbf{y} \\ L_{2}{ }^{\mathrm{T}} & \mathbf{y}^{\mathrm{T}} & L_{3}\end{array}\right)$, where $L_{1} \in \mathbb{R}^{(i-1) \times(i-1)}, L_{3} \in \mathbb{R}^{(n-i) \times(n-i)}, \mathbf{x} \in \mathbb{R}^{i-1}, \mathbf{y}^{\mathrm{T}} \in \mathbb{R}^{n-i}$. Suppose $\mathcal{L}_{G}(i)^{-1}=\left(\begin{array}{cc}\widetilde{L_{1}} & \widetilde{L_{2}} \\ \widetilde{L_{2}} & \widetilde{L_{3}}\end{array}\right)$, where $\widetilde{L_{1}} \in \mathbb{R}^{(i-1) \times(i-1)}, \widetilde{L_{3}} \in \mathbb{R}^{(n-i) \times(n-i)}$. Then $\left(\begin{array}{ccc}\widetilde{L_{1}} & \mathbf{0} & \widetilde{L_{2}} \\ \mathbf{0} & 0 & \mathbf{0} \\ \widetilde{L_{2}} & \mathbf{0} & \widetilde{L_{3}}\end{array}\right)$ is a symmetric $\{1\}$-inverse of $\mathcal{L}_{G}$.

\section{Inverse Perron values of uniform hypergraphs}

In the following theorem, the relationship between inverse Perron values and connectivity of a hypergraph is presented.

Theorem 6. Let $\mathcal{G}$ be a k-uniform hypergraph. Then the following statements are equivalent:

(1) $\mathcal{G}$ is connected.

(2) $\alpha_{j}(\mathcal{G})>0$ for all $j \in V(\mathcal{G})$.

(3) $\alpha_{j}(\mathcal{G})>0$ for some $j \in V(\mathcal{G})$.

Proof. $(1) \Longrightarrow(2)$. If $\mathcal{G}$ is connected, then by Lemma 1 , we know that $\alpha_{j}(\mathcal{G})>0$ for all $j \in V(\mathcal{G})$.

$(2) \Longrightarrow(3)$. Obviously.

$(3) \Longrightarrow(1)$. Suppose that $\mathcal{G}$ is disconnected. For any $j \in V(\mathcal{G})$, let $\mathcal{G}_{1}$ be the component of $\mathcal{G}$ such that $j \notin V\left(\mathcal{G}_{1}\right)$. Let $\mathbf{x}=\left(x_{1}, \ldots, x_{|V(\mathcal{G})|}\right)^{\mathrm{T}}$ be the vector satisfying

$$
x_{i}= \begin{cases}\left|V\left(\mathcal{G}_{1}\right)\right|^{-\frac{1}{k}}, & \text { if } i \in V\left(\mathcal{G}_{1}\right), \\ 0, & \text { otherwise. }\end{cases}
$$

Clearly, we have $\sum_{i=1}^{n} x_{i}^{k}=1$. Then we have $0 \leqslant \alpha_{j}(\mathcal{G}) \leqslant \mathcal{L}_{\mathcal{G}} \mathbf{x}^{k}=0$ for any $j \in V(\mathcal{G})$, a contradiction to (3). Hence $\mathcal{G}$ is connected if (3) holds. 
The bipartition width of a hypergraph $\mathcal{G}$ is defined as [18, 28]

$$
\operatorname{bw}(\mathcal{G})=\min \left\{|E(S, \bar{S})|: S \subseteq V(\mathcal{G}),|S|=\left\lfloor\frac{n}{2}\right\rfloor\right\}
$$

where $\left\lfloor\frac{n}{2}\right\rfloor$ denotes the maximum integer not larger than $\frac{n}{2}$. The computation of $\operatorname{bw}(\mathcal{G})$ is very difficult even for the graph case. In [22], Mohar and Poljak used the algebraic connectivity to obtain a lower bound on the bipartition width of a graph. In the following theorem, we use the inverse Perron values to obtain a lower bound on the bipartition width of a uniform hypergraph.

Theorem 7. Let $\mathcal{G}$ be a $k$-uniform hypergraph with $n$ vertices. Then

$$
\mathrm{bw}(\mathcal{G}) \geqslant \frac{n+(-1)^{n}}{k(n+1)} \sum_{j=1}^{n} \alpha_{j}(\mathcal{G}) .
$$

Proof. Suppose that $S_{0} \subseteq V(\mathcal{G})$ satisfying $\left|S_{0}\right|=\left\lfloor\frac{n}{2}\right\rfloor$ and $\left|E\left(S_{0}, \overline{S_{0}}\right)\right|=\operatorname{bw}(\mathcal{G})$. Let $\mathbf{x}=\left(x_{1}, \ldots, x_{n}\right)^{\mathrm{T}}$ be the vector satisfying

$$
x_{i}= \begin{cases}\left|S_{0}\right|^{-\frac{1}{k}}, & i \in S_{0}, \\ 0, & i \in \overline{S_{0}} .\end{cases}
$$

Then $\sum_{i=1}^{n} x_{i}^{k}=1$. For $j \in \overline{S_{0}}$, we get

$$
\begin{aligned}
\alpha_{j}(\mathcal{G}) \leqslant & \mathcal{L}_{\mathcal{G}} \mathbf{x}^{k}=\sum_{\left\{i_{1}, \ldots, i_{k}\right\} \in E(\mathcal{G})}\left(x_{i_{1}}^{k}+\cdots+x_{i_{k}}^{k}-k x_{i_{1}} \cdots x_{i_{k}}\right) \\
\alpha_{j}(\mathcal{G}) \leqslant & \sum_{\left\{i_{1}, \ldots, i_{k}\right\} \in E\left(S_{0}, \overline{S_{0}}\right)}\left(x_{i_{1}}^{k}+\cdots+x_{i_{k}}^{k}-k x_{i_{1}} \cdots x_{i_{k}}\right) \\
& =\frac{1}{\left|S_{0}\right|} \sum_{e \in E\left(S_{0}, \overline{S_{0}}\right)}\left|e \cap S_{0}\right|=\frac{t\left(S_{0}\right) \mathrm{bw}(\mathcal{G})}{\left|S_{0}\right|},
\end{aligned}
$$

where $t\left(S_{0}\right)=\frac{1}{\left|E\left(S_{0}, \overline{S_{0}}\right)\right|} \sum_{e \in E\left(S_{0}, \overline{S_{0}}\right)}\left|e \cap S_{0}\right|$.

Similarly, for $j \in S_{0}$, we obtain

$$
\alpha_{j}(\mathcal{G}) \leqslant \frac{\left(k-t\left(S_{0}\right)\right) \operatorname{bw}(\mathcal{G})}{\left|\overline{S_{0}}\right|} .
$$

Combining (1) and (2), we get

$$
\sum_{j=1}^{n} \alpha_{j}(\mathcal{G})=\sum_{j \in S_{0}} \alpha_{j}(\mathcal{G})+\sum_{j \in \overline{S_{0}}} \alpha_{j}(\mathcal{G}) \leqslant \frac{\left|S_{0}\right|\left(k-t\left(S_{0}\right)\right) \mathrm{bw}(\mathcal{G})}{\left|\overline{S_{0}}\right|}+\frac{\left|\overline{S_{0}}\right| t\left(S_{0}\right) \mathrm{bw}(\mathcal{G})}{\left|S_{0}\right|} .
$$


If $n$ is even, then $\left|S_{0}\right|=\left|\overline{S_{0}}\right|$ and $\operatorname{bw}(\mathcal{G}) \geqslant \frac{1}{k} \sum_{j=1}^{n} \alpha_{j}(\mathcal{G})$. If $n$ is odd, then $\left|S_{0}\right|=\left|\overline{S_{0}}\right|-1=$ $\frac{n-1}{2}$ and

$$
\sum_{j=1}^{n} \alpha_{j}(\mathcal{G}) \leqslant k \frac{\left|\overline{S_{0}}\right|}{\left|S_{0}\right|} \operatorname{bw}(\mathcal{G})=\frac{k(n+1) \mathrm{bw}(\mathcal{G})}{n-1}, \operatorname{bw}(\mathcal{G}) \geqslant \frac{n-1}{k(n+1)} \sum_{j=1}^{n} \alpha_{j}(\mathcal{G}) .
$$

The isoperimetric number of a $k$-uniform hypergraph $\mathcal{G}$ is defined as

$$
i(\mathcal{G})=\min \left\{\frac{|E(S, \bar{S})|}{|S|}: S \subseteq V(\mathcal{G}), 0<|S| \leqslant \frac{|V(\mathcal{G})|}{2}\right\}
$$

Let $\beta(\mathcal{G})=\max _{j \in V(\mathcal{G})} \alpha_{j}(\mathcal{G})$ denote the maximum inverse Perron value of $\mathcal{G}$. In [20], it was shown that $i(\mathcal{G}) \geqslant \frac{2}{k} \alpha(\mathcal{G})$. We improve it as follows.

Theorem 8. Let $\mathcal{G}$ be a k-uniform hypergraph. Then

$$
i(\mathcal{G}) \geqslant \frac{\alpha(\mathcal{G})+\beta(\mathcal{G})}{k} .
$$

Proof. Suppose that $S_{1} \subseteq V(\mathcal{G})$ satisfying $0<\left|S_{1}\right| \leqslant \frac{|V(\mathcal{G})|}{2}$ and $\frac{\left|E\left(S_{1}, \overline{S_{1}}\right)\right|}{\left|S_{1}\right|}=i(\mathcal{G})$. Let $\mathbf{x}=\left(x_{1}, \ldots, x_{n}\right)^{\mathrm{T}}$ be the vector satisfying

$$
x_{i}= \begin{cases}\left|S_{1}\right|^{-\frac{1}{k}}, & i \in S_{1}, \\ 0, & i \in \overline{S_{1}} .\end{cases}
$$

Then $\sum_{i=1}^{n} x_{i}^{k}=1$. For $j \in \overline{S_{1}}$, we obtain

$$
\alpha_{j}(\mathcal{G}) \leqslant \mathcal{L}_{\mathcal{G}} \mathbf{x}^{k}=\frac{t\left(S_{1}\right)\left|E\left(S_{1}, \overline{S_{1}}\right)\right|}{\left|S_{1}\right|}=t\left(S_{1}\right) i(\mathcal{G}),
$$

where $t\left(S_{1}\right)=\frac{1}{\left|E\left(S_{1}, \overline{S_{1}}\right)\right|} \sum_{e \in E\left(S_{1}, \overline{S_{1}}\right)}\left|e \cap S_{1}\right|$.

Similarly, for $j \in S_{1}$, we get

$$
\alpha_{j}(\mathcal{G}) \leqslant \frac{\left(k-t\left(S_{1}\right)\right)\left|E\left(S_{1}, \overline{S_{1}}\right)\right|}{\left|\overline{S_{1}}\right|} \leqslant\left(k-t\left(S_{1}\right)\right) i(\mathcal{G}) .
$$

Let $\alpha_{s}(\mathcal{G})=\beta(\mathcal{G})$. If $s \in \overline{S_{1}}$, by $(3)$, we get

$$
\beta(\mathcal{G})=\alpha_{s}(\mathcal{G}) \leqslant t\left(S_{1}\right) i(\mathcal{G}) .
$$

From (4), we have

$$
\alpha(\mathcal{G})=\min _{j \in V(\mathcal{G})} \alpha_{j}(\mathcal{G}) \leqslant \min _{j \in S_{1}} \alpha_{j}(\mathcal{G}) \leqslant\left(k-t\left(S_{1}\right)\right) i(\mathcal{G})
$$


Then

$$
\alpha(\mathcal{G})+\beta(\mathcal{G}) \leqslant t\left(S_{1}\right) i(\mathcal{G})+\left(k-t\left(S_{1}\right)\right) i(\mathcal{G})=k i(\mathcal{G}) .
$$

Similarly, if $s \in S_{1}$, we can also obtain $\alpha(\mathcal{G})+\beta(\mathcal{G}) \leqslant k i(\mathcal{G})$.

From the above discussion, we get $i(\mathcal{G}) \geqslant \frac{\alpha(\mathcal{G})+\beta(\mathcal{G})}{k}$.

The distance $d(u, v)$ between two distinct vertices $u$ and $v$ of $\mathcal{G}$ is the length of the shortest path connecting them. The eccentricity of a vertex $v$ is $\operatorname{ecc}(v)=\max \{d(u, v)$ : $u \in V(\mathcal{G})\}$. The diameter and radius of $\mathcal{G}$ are defined as $\operatorname{diam}(\mathcal{G})=\max _{v \in V(\mathcal{G})} \operatorname{ecc}(v)$ and $\operatorname{rad}(\mathcal{G})=\min _{v \in V(\mathcal{G})} \operatorname{ecc}(v)$, respectively.

Theorem 9. Let $\mathcal{G}$ be a connected $k$-uniform hypergraph with $n$ vertices. Then

$$
\operatorname{ecc}(j) \geqslant \frac{k}{2(k-1)(n-1) \alpha_{j}(\mathcal{G})}, j \in V(\mathcal{G}) .
$$

Proof. For $j \in V(\mathcal{G})$, let $\mathbf{x}=\left(x_{1}, \ldots, x_{n}\right)^{\mathrm{T}} \in \mathbb{R}_{+}^{n}$ satisfying $x_{j}=0, \sum_{i=1}^{n} x_{i}^{k}=1$ and $\alpha_{j}(\mathcal{G})=\mathcal{L}_{\mathcal{G}} \mathbf{x}^{k}$. Then

$$
\alpha_{j}(\mathcal{G})=\mathcal{L}_{\mathcal{G}} \mathbf{x}^{k}=\sum_{\left\{i_{1}, \ldots, i_{k}\right\} \in E(\mathcal{G})}\left(x_{i_{1}}^{k}+\cdots+x_{i_{k}}^{k}-k x_{i_{1}} \cdots x_{i_{k}}\right)
$$

From AM-GM inequality, it yields that

$$
\sum_{1 \leqslant s<t \leqslant k} x_{i_{s}}^{\frac{k}{2}} x_{i_{t}}^{\frac{k}{2}} \geqslant \frac{k(k-1)}{2}\left(\prod_{1 \leqslant s<t \leqslant k} x_{i_{s}}^{\frac{k}{2}} x_{i_{t}}^{\frac{k}{2}}\right)^{\frac{2}{k(k-1)}}=\frac{k(k-1)}{2} x_{i_{1}} \cdots x_{i_{k}} .
$$

By (5) and (6), we have

$$
\begin{aligned}
\alpha_{j}(\mathcal{G}) & \geqslant \sum_{\left\{i_{1}, \ldots, i_{k}\right\} \in E(\mathcal{G})}\left(x_{i_{1}}^{k}+\cdots+x_{i_{k}}^{k}-\frac{2}{k-1} \sum_{1 \leqslant s<t \leqslant k} x_{i_{s}}^{\frac{k}{2}} x_{i_{t}}^{\frac{k}{2}}\right) \\
& =\frac{1}{k-1} \sum_{\left\{i_{1}, \ldots, i_{k}\right\} \in E(\mathcal{G})} \sum_{1 \leqslant s<t \leqslant k}\left(x_{i_{s}}^{\frac{k}{2}}-x_{i_{t}}^{\frac{k}{2}}\right)^{2} \\
& =\frac{1}{k-1} \sum_{e \in E(\mathcal{G})} \sum_{s, t \in e}\left(x_{s}^{\frac{k}{2}}-x_{t}^{\frac{k}{2}}\right)^{2} .
\end{aligned}
$$

Let $v_{0} \in\left\{i \mid x_{i}=\max _{p \in V(\mathcal{G})} x_{p}\right\}$. Let $\mathcal{P}=v_{0} e_{1} v_{1} e_{2} \cdots v_{l-1} e_{l} v_{l}$ be the shortest path from vertex $v_{0}$ to vertex $v_{l}=j$. Then $x_{v_{0}}^{k} \geqslant \frac{1}{n-1}, x_{v_{l}}=0$ and

$$
\sum_{e \in E(\mathcal{G})} \sum_{s, t \in e}\left(x_{s}^{\frac{k}{2}}-x_{t}^{\frac{k}{2}}\right)^{2} \geqslant \sum_{e \in E(\mathcal{P})} \sum_{s, t \in e}\left(x_{s}^{\frac{k}{2}}-x_{t}^{\frac{k}{2}}\right)^{2}
$$




$$
\begin{aligned}
& \geqslant \sum_{i=1}^{l}\left(\left(x_{v_{i-1}}^{\frac{k}{2}}-x_{v_{i}}^{\frac{k}{2}}\right)^{2}+\sum_{u_{j} \in e_{i} \backslash\left\{v_{i-1}, v_{i}\right\}}\left(\left(x_{v_{i-1}}^{\frac{k}{2}}-x_{u_{j}}^{\frac{k}{2}}\right)^{2}+\left(x_{u_{j}}^{\frac{k}{2}}-x_{v_{i}}^{\frac{k}{2}}\right)^{2}\right)\right) \\
& \geqslant \sum_{i=1}^{l}\left(\left(x_{v_{i-1}}^{\frac{k}{2}}-x_{v_{i}}^{\frac{k}{2}}\right)^{2}+\frac{1}{2} \sum_{u_{j} \in e_{i} \backslash\left\{v_{i-1}, v_{i}\right\}}\left(x_{v_{i-1}}^{\frac{k}{2}}-x_{u_{j}}^{\frac{k}{2}}+x_{u_{j}}^{\frac{k}{2}}-x_{v_{i}}^{\frac{k}{2}}\right)^{2}\right)^{2} \\
& =\sum_{i=1}^{l}\left(\left(x_{v_{i-1}}^{\frac{k}{2}}-x_{v_{i}}^{\frac{k}{2}}\right)^{2}+\frac{k-2}{2}\left(x_{v_{i-1}}^{\frac{k}{2}}-x_{v_{i}}^{\frac{k}{2}}\right)^{2}\right) \\
& =\frac{k}{2} \sum_{i=1}^{l}\left(x_{v_{i-1}}^{\frac{k}{2}}-x_{v_{i}}^{\frac{k}{2}}\right)^{2} .
\end{aligned}
$$

By Cauchy-Schwarz inequality, we obtain

$$
\begin{aligned}
& \sum_{e \in E(\mathcal{G})} \sum_{s, t \in e}\left(x_{s}^{\frac{k}{2}}-x_{t}^{\frac{k}{2}}\right)^{2} \geqslant \frac{k}{2} \sum_{i=1}^{l}\left(x_{v_{i-1}}^{\frac{k}{2}}-x_{v_{i}}^{\frac{k}{2}}\right)^{2} \geqslant \frac{k}{2 l}\left(\sum_{i=1}^{l}\left(x_{v_{i-1}}^{\frac{k}{2}}-x_{v_{i}}^{\frac{k}{2}}\right)\right)^{2} \\
= & \frac{k}{2 l}\left(x_{v_{0}}^{\frac{k}{2}}-x_{v_{l}}^{\frac{k}{2}}\right)^{2} \geqslant \frac{k}{2 \operatorname{ecc}(j)}\left(x_{v_{0}}^{\frac{k}{2}}-x_{v_{l}}^{\frac{k}{2}}\right)^{2} \geqslant \frac{k}{2(n-1) \operatorname{ecc}(j)} .
\end{aligned}
$$

From (7) and (8), it yields that

$$
\alpha_{j}(\mathcal{G}) \geqslant \frac{k}{2(k-1)(n-1) \operatorname{ecc}(j)}, \operatorname{ecc}(j) \geqslant \frac{k}{2(k-1)(n-1) \alpha_{j}(\mathcal{G})} .
$$

For a connected $k$-uniform hypergraph $\mathcal{G}$ with $n$ vertices, [20] showed that

$$
\operatorname{diam}(\mathcal{G}) \geqslant \frac{4}{n^{2}(k-1) \alpha(\mathcal{G})} .
$$

By Theorem 9, we obtain the following improved result.

Corollary 10. Let $\mathcal{G}$ be a connected $k$-uniform hypergraph with $n$ vertices. Then

$$
\operatorname{diam}(\mathcal{G}) \geqslant \frac{k}{2(k-1)(n-1) \alpha(\mathcal{G})}, \operatorname{rad}(\mathcal{G}) \geqslant \frac{k}{2(k-1)(n-1) \beta(\mathcal{G})} .
$$

In [27], it was shown that $\alpha(\mathcal{G}) \leqslant \delta$, where $\delta$ is the minimum degree of $\mathcal{G}$. We improve it as follows.

Theorem 11. Let $\mathcal{G}$ be a $k$-uniform hypergraph with $n$ vertices. Then

$$
\alpha_{j}(\mathcal{G}) \leqslant \frac{(k-1) d_{j}}{n-1}, j \in V(\mathcal{G})
$$


Proof. For $j \in V(\mathcal{G})$, let $\mathbf{x}=\left(x_{1}, \ldots, x_{n}\right)^{\mathrm{T}}$ be the vector satisfying

$$
x_{i}= \begin{cases}(n-1)^{-\frac{1}{k}}, & i \neq j, \\ 0, & i=j .\end{cases}
$$

Then $\sum_{i=1}^{n} x_{i}^{k}=1$, and we get

$$
\begin{aligned}
\alpha_{j}(\mathcal{G}) & \leqslant \mathcal{L}_{\mathcal{G}} \mathbf{x}^{k}=\sum_{\left\{i_{1}, \ldots, i_{k}\right\} \in E(\mathcal{G})}\left(x_{i_{1}}^{k}+\cdots+x_{i_{k}}^{k}-k x_{i_{1}} \cdots x_{i_{k}}\right) \\
& =\sum_{\left\{i_{1}, \ldots, i_{k}\right\} \in E_{j}(\mathcal{G})}\left(x_{i_{1}}^{k}+\cdots+x_{i_{k}}^{k}\right)=\frac{(k-1) d_{j}}{n-1}
\end{aligned}
$$

where $E_{j}(\mathcal{G})$ denotes the set of edges containing $j$.

By Theorem 11, we obtain the following result.

Corollary 12. Let $\mathcal{G}$ be a $k$-uniform hypergraph with $n$ vertices and $m$ edges. Then

$$
\sum_{j=1}^{n} \alpha_{j}(\mathcal{G}) \leqslant \frac{(k-1) k m}{n-1}, j \in V(\mathcal{G}) .
$$

Let $\mathcal{G}$ be a $k$-uniform hypergraph. For $x, y \in V(\mathcal{G})$, let $c(x, y)=\mid\{e \in E(\mathcal{G}): x, y \in$ $e\} \mid$. A 2 - $(n, b, k, r, \lambda)$ design can be regarded as a $k$-uniform $r$-regular hypergraph $\mathcal{G}$ on $n$ vertices, $b$ edges, and $c(x, y)=\lambda$ for any pair of distinct $x, y \in V(\mathcal{G})$. A 2-design satisfying $n=b$ is called a symmetric design.

Theorem 13. Let $\mathcal{G}$ be a connected $k$-uniform hypergraph with $n$ vertices. Then $\mathcal{G}$ is a 2-design if and only if $\alpha_{1}(\mathcal{G})=\cdots=\alpha_{n}(\mathcal{G})=\frac{\Delta(k-1)}{n-1}$, where $\Delta$ is the maximum degree of $\mathcal{G}$.

Proof. We first prove the necessity. If $\mathcal{G}$ is a $2-(n, b, k, r, \lambda)$ design, then $\lambda(n-1)=r(k-1)$ and $\Delta=r=d_{1}=\cdots=d_{n}$. For any $j \in V(\mathcal{G})$, by Theorem 11, we have

$$
\alpha_{j}(\mathcal{G}) \leqslant \frac{r(k-1)}{n-1}=\lambda .
$$

Let $\mathbf{x}=\left(x_{1}, \ldots, x_{n}\right)^{\mathrm{T}} \in \mathbb{R}_{+}^{n}$ satisfying $x_{j}=0, \sum_{i=1}^{n} x_{i}^{k}=1$ and $\alpha_{j}(\mathcal{G})=\mathcal{L}_{\mathcal{G}} \mathbf{x}^{k}$. Then we get

$$
\alpha_{j}(\mathcal{G})=\mathcal{L}_{\mathcal{G}} \mathbf{x}^{k} \geqslant \sum_{\left\{i_{1}, \ldots, i_{k}\right\} \in E_{j}(\mathcal{G})}\left(x_{i_{1}}^{k}+\cdots+x_{i_{k}}^{k}-k x_{i_{1}} \cdots x_{i_{k}}\right)=\lambda \sum_{i \neq j} x_{i}^{k}=\lambda .
$$

Combining (9) and (10), we get

$$
\alpha_{1}(\mathcal{G})=\cdots=\alpha_{n}(\mathcal{G})=\lambda=\frac{r(k-1)}{n-1}=\frac{\Delta(k-1)}{n-1} .
$$


Next we prove the sufficiency. Let $\alpha_{1}(\mathcal{G})=\cdots=\alpha_{n}(\mathcal{G})=\frac{\Delta(k-1)}{n-1}$. From Theorem 11, we obtain $d_{1}=\cdots=d_{n}=\Delta$. Let $\mathbf{z}=\left((n-1)^{-\frac{1}{k}}, \ldots,(n-1)^{-\frac{1}{k}}\right)^{\mathrm{T}} \in \mathbb{R}_{+}^{n-1}$. For $j \in V(\mathcal{G})$, let $\mathbf{y}=\left(y_{1}, \ldots, y_{n}\right)^{\mathrm{T}} \in \mathbb{R}_{+}^{n}$ be a vector such that $y_{i}=0$ if $i=j$ and $y_{i}=(n-1)^{-\frac{1}{k}}$ otherwise. Then

$$
\begin{aligned}
\mathcal{L}_{\mathcal{G}} \mathbf{y}^{k} & =\sum_{\left\{i_{1}, \ldots, i_{k}\right\} \in E(\mathcal{G})}\left(y_{i_{1}}^{k}+\cdots+y_{i_{k}}^{k}-k y_{i_{1}} \cdots y_{i_{k}}\right)=\sum_{\left\{i_{1}, \ldots, i_{k}\right\} \in E_{j}(\mathcal{G})}\left(y_{i_{1}}^{k}+\cdots+y_{i_{k}}^{k}\right) \\
& =\frac{\Delta(k-1)}{n-1}=\alpha_{j}(\mathcal{G})=\alpha(\mathcal{G}) .
\end{aligned}
$$

We know that $\alpha(\mathcal{G})=\alpha_{j}(\mathcal{G})$ is the smallest H-eigenvalue of $\mathcal{L}_{\mathcal{G}}(j)$. Since $\mathcal{L}_{\mathcal{G}}(j) \mathbf{z}^{k}=$ $\mathcal{L}_{\mathcal{G}} \mathbf{y}^{k}=\alpha(\mathcal{G}), \mathbf{z}$ is an H-eigenvector corresponding to $\alpha(\mathcal{G})$, that is

$$
\alpha(\mathcal{G}) \mathbf{z}^{[k-1]}=\mathcal{L}_{\mathcal{G}}(j) \mathbf{z}^{k-1}
$$

For all $i \in V(\mathcal{G}) \backslash\{j\}$, we have

$$
\begin{aligned}
\alpha(\mathcal{G}) & =\frac{1}{z_{i}^{k-1}}\left(\mathcal{L}_{\mathcal{G}}(j) \mathbf{z}^{k-1}\right)_{i}=\frac{1}{z_{i}{ }^{k-1}} \sum_{i_{2}, \ldots i_{k} \neq j}\left(\mathcal{L}_{\mathcal{G}}(j)\right)_{i i_{2} \cdots i_{k}} z_{i_{2}} \cdots z_{i_{k}} \\
& =\sum_{i_{2}, \ldots, i_{k} \neq j}\left(\mathcal{L}_{\mathcal{G}}\right)_{i i_{2} \cdots i_{k}}=c(i, j) .
\end{aligned}
$$

So $c(i, j)=\alpha(\mathcal{G})$ for any pair of distinct $i, j \in V(\mathcal{G})$, which implies that $\mathcal{G}$ is a 2-design.

We give an estimation of the edge connectivity of a 2-design as follows.

Theorem 14. Let $\mathcal{G}$ be a $2-(n, b, k, r, \lambda)$ design. Then

$$
\frac{n \lambda}{k} \leqslant e(\mathcal{G}) \leqslant \frac{(n-1) \lambda}{k-1} .
$$

Moreover, if $\mathcal{G}$ is a symmetric design, then $e(\mathcal{G})=k=r$.

Proof. Since $\mathcal{G}$ is a $2-(n, b, k, r, \lambda)$ design, we have $\lambda(n-1)=r(k-1)$. By Theorem 13, we have

$$
\alpha(\mathcal{G})=\frac{r(k-1)}{n-1}=\lambda .
$$

It follows from Lemma 2 that

$$
\frac{n \lambda}{k}=\frac{n}{k} \alpha(\mathcal{G}) \leqslant e(\mathcal{G}) \leqslant r=\frac{(n-1) \lambda}{k-1} .
$$

Moreover, if $\mathcal{G}$ is a symmetric design, then $n=b$. Since $n r=b k$, we have $r=k$. From $\lambda(n-1)=r(k-1)$ and (11), we have

$$
\frac{n(k-1)}{n-1} \leqslant e(\mathcal{G}) \leqslant k .
$$

Since $e(\mathcal{G})$ is a positive integer, we get $e(\mathcal{G})=k=r$. 


\section{Inverse Perron values and resistance distance of graphs}

For a vertex $i$ of a connected graph $G$, we define its resistance eccentricity as $r_{i}(G)=$ $\max _{j \in V(G)} r_{i j}$

Theorem 15. Let $G$ be a connected graph. For any $i \in V(G)$, we have

$$
r_{i}(G) \leqslant \frac{1}{\alpha_{i}(G)}
$$

Proof. Without loss of generality, assume that $i$ is the vertex corresponding to the last row of the Laplacian matrix $\mathcal{L}_{G}$. Since $\alpha_{i}(G)$ is the minimum eigenvalue of the principal submatrix $\mathcal{L}_{G}(i), \alpha_{i}^{-1}(G)$ is the spectral radius of the symmetric nonnegative matrix $\mathcal{L}_{G}(i)^{-1}$. So $\alpha_{i}^{-1}(G) \geqslant \max _{j \neq i}\left(\mathcal{L}_{G}(i)^{-1}\right)_{j j}$.

By Lemmas 5 and 3 , we get $r_{i j}(G)=\left(\mathcal{L}_{G}(i)^{-1}\right)_{j j}$ for any $j \neq i$. Hence

$$
\begin{aligned}
\alpha_{i}^{-1}(G) & \geqslant \max _{j \neq i}\left(\mathcal{L}_{G}(i)^{-1}\right)_{j j}=r_{i}(G), \\
r_{i}(G) & \leqslant \frac{1}{\alpha_{i}(G)} .
\end{aligned}
$$

For a vertex $i$ of a connected graph $G$, its resistance centrality is defined as $K f_{i}(G)=$ $\sum_{j \in V(G)} r_{i j}(G)$. It is used to measure the centrality of a network [4]. Note that $K f(G)=$ $\sum_{\{i, j\} \subseteq V(G)} r_{i j}(G)=\frac{1}{2} \sum_{i \in V(G)} K f_{i}(G)$.

Theorem 16. Let $G$ be a connected graph with $n$ vertices. For any $i \in V(G)$, we have

$$
n K f_{i}(G)-K f(G) \leqslant \frac{n-1}{\alpha_{i}(G)}
$$

Proof. Note that $\alpha_{i}^{-1}(G)$ is the maximum eigenvalue of the symmetric matrix $\mathcal{L}_{G}(i)^{-1}$. Let $\mathbf{e}$ be the all-ones column vector, then

$$
\alpha_{i}^{-1}(G) \geqslant \frac{\mathbf{e}^{\top} \mathcal{L}_{G}(i)^{-1} \mathbf{e}}{\mathbf{e}^{\top} \mathbf{e}}=\frac{\mathbf{e}^{\top} \mathcal{L}_{G}(i)^{-1} \mathbf{e}}{n-1} .
$$

By Lemmas 5 and 4, we have

$$
K f(G)=n \operatorname{tr}\left(\mathcal{L}_{G}(i)^{-1}\right)-\mathbf{e}^{\top} \mathcal{L}_{G}(i)^{-1} \mathbf{e} .
$$

From Lemmas 5 and 3 , we get $r_{i j}(G)=\left(\mathcal{L}_{G}(i)^{-1}\right)_{j j}$ for any $j \neq i$. Hence $\operatorname{tr}\left(\mathcal{L}_{G}(i)^{-1}\right)=$ $K f_{i}(G)$ and

$$
K f(G)=n K f_{i}(G)-\mathbf{e}^{\top} \mathcal{L}_{G}(i)^{-1} \mathbf{e}
$$


By $\alpha_{i}^{-1}(G) \geqslant \frac{\mathbf{e}^{\top} \mathcal{L}_{G}(i)^{-1} \mathbf{e}}{n-1}$ we get

$$
\begin{aligned}
\alpha_{i}^{-1}(G) \geqslant \frac{\mathbf{e}^{\top} \mathcal{L}_{G}(i)^{-1} \mathbf{e}}{n-1} & =\frac{n K f_{i}(G)-K f(G)}{n-1}, \\
n K f_{i}(G)-K f(G) & \leqslant \frac{n-1}{\alpha_{i}(G)} .
\end{aligned}
$$

Corollary 17. Let $G$ be a connected graph with $n$ vertices. Then

$$
K f(G) \leqslant \frac{n-1}{n} \sum_{i=1}^{n} \alpha_{i}^{-1}(G) .
$$

Proof. By Theorem 16, we have

$$
\begin{gathered}
\sum_{i=1}^{n} \frac{n-1}{\alpha_{i}(G)} \geqslant \sum_{i=1}^{n}\left(n K f_{i}(G)-K f(G)\right)=n K f(G), \\
K f(G) \leqslant \frac{n-1}{n} \sum_{i=1}^{n} \alpha_{i}^{-1}(G) .
\end{gathered}
$$

\section{References}

[1] E. Andrade and G. Dahl. Combinatorial Perron values of trees and bottleneck matrices. Linear Multilinear Algebra, 65:2387-2405, 2017.

[2] R. B. Bapat. Graphs and Matrices. Springer, London, 2010.

[3] E. Bendito, A. Carmona, A. M. Encinas and J. M. Gesto. A formula for the Kirchhoff index. Int. J. Quantum Chem., 108:1200-1206, 2008.

[4] E. Bozzo and M. Franceschet. Resistance distance, closeness, and betweenness. Social Networks, 35:460-469, 2013.

[5] A. Bretto. Hypergraph Theory: An Introduction. Springer, New York, 2013.

[6] C. Bu, B. Yan, X. Zhou and J. Zhou. Resistance distance in subdivision-vertex join and subdivision-edge join of graphs. Linear Algebra Appl., 458:454-462, 2014.

[7] C. Bu, J. Zhou and Y. Wei. E-cospectral hypergraphs and some hypergraphs determined by their spectra. Linear Algebra Appl., 459:397-403, 2014.

[8] J. Cooper and A. Dutle. Spectra of uniform hypergraphs. Linear Algebra Appl., 436:3268-3292, 2012.

[9] C. Cui, Z. Luo, L. Qi and H. Yan. Computing the analytic connectivity of a uniform hypergraph. arXiv:1611.01372v1, 2016.

[10] Y. Fan, Y. Tan, X. Peng and A. Liu. Maximizing spectral radii of uniform hypergraphs with few edges. Discuss. Math. Graph Theory, 36:845-856, 2016. 
[11] M. Fiedler. Algebraic connectivity of graphs. Czech. Math. J., 23:298-305, 1973.

[12] A. Ghosh, S. Boyd and A. Saberi. Minimizing effective resistance of a graph. SIAM Rev., 50:37-66, 2008.

[13] S. Hu and L. Qi. The eigenvectors associated with the zero eigenvalues of the Laplacian and signless Laplacian tensors of a uniform hypergraph. Discrete Appl. Math., 169:140-151, 2014.

[14] M. Khan, Y. Fan and Y. Tan. The H-spectra of a class of generalized power hypergraphs. Discrete Math., 339:1682-1689, 2016.

[15] S. Kirkland, M. Neumann and B. L. Shader. Characteristic vertices of weighted trees via Perron values. Linear Multilinear Algebra, 40:311-325, 1996.

[16] S. Kirkland, M. Neumann and B. L. Shader. Distances in weighted trees and group inverse of Laplacian matrices. SIAM J. Matrix Anal. Appl., 18:827-841, 1997.

[17] D. J. Klein and M. Randić. Resistance distance. J. Math. Chem., 12:81-95, 1993.

[18] G. Li, L. Qi and G. Yu. The Z-eigenvalues of a symmetric tensor and its application to spectral hypergraph theory. Numer. Linear Algebra Appl., 20:1001-1029, 2013.

[19] H. Li, J. Shao and L. Qi. The extremal spectral radii of $k$-uniform supertrees. $J$. Comb. Optim., 32 :741-764, 2016.

[20] W. Li, J. Cooper and A. Chang. Analytic connectivity of $k$-uniform hypergraphs. Linear Multilinear Algebra, 65:1247-1259, 2017.

[21] L.H. Lim. Singular values and eigenvalues of tensors: a variational approach. In Proc. IEEE Int. Workshop on Comput. Advances in Multi-Sensor Adaptive Processing (CAMSAP' 05), 1:129-132, 2005.

[22] B. Mohar and S. Poljak. Eigenvalues and the max-cut problem. Czech. Math. J., 40:343-352, 1990.

[23] A. Nikseresht and Z. Sepasdar. On the Kirchhoff and the Wiener indices of graphs and block decomposition. Electron. J. Combin., 21(1):\#P1.25, 2014.

[24] K. Pearson and T. Zhang. On spectral hypergraph theory of the adjacency tensor. Graph. Combin., 30:1233-1248, 2014.

[25] K. Pearson and T. Zhang. The Laplacian tensor of a multi-hypergraph. Discrete Math., 338:972-982, 2015.

[26] L. Qi. Eigenvalues of a real supersymmetric tensor. J. Symb. Comput., 40:1302-1324, 2005.

[27] L. Qi. $H^{+}$-eigenvalues of Laplacian and signless Laplacian tensors. Commun. Math. Sci. 12:1045-1064, 2014.

[28] J. A. Rodríguez. Laplacian eigenvalues and partition problems in hypergraphs. Appl. Math. Lett., 22:916-921, 2009.

[29] J. Shao and X. Yuan. Some properties of the Laplace and normalized Laplace spectra of uniform hypergraphs. Linear Algebra Appl., 531:98-117, 2017. 
[30] L. Sun, W. Wang, J. Zhou and C. Bu. Some results on resistance distances and resistance matrices. Linear Multilinear Algebra, 63:523-533, 2015.

[31] Y. Yang and D. J. Klein. Resistance distance-based graph invariants of subdivisions and triangulations of graphs. Discrete Appl. Math., 181:260-274, 2015.

[32] L. Zhang, L. Qi and G. Zhou. M-tensors and some applications. SIAM J. Matrix Anal. Appl., 35:437-452, 2014.

[33] B. Zhou and N. Trinajstić. On resistance-distance and Kirchhoff index. J. Math. Chem., 46:283-289, 2009.

[34] J. Zhou, L. Sun and C. Bu. Resistance characterizations of equiarboreal graphs. Discrete Math., 340:2864-2870, 2017.

[35] J. Zhou, L. Sun, W. Wang and C. Bu. Some spectral properties of uniform hypergraphs. Electron. J. Combin., 21(4):\#P4.24, 2014.

[36] J. Zhou, Z. Wang and C. Bu. On the resistance matrix of a graph. Electron. J. Combin. 23(1):\#P1.41, 2016. 